\title{
A Modified Method to Solve the One-Dimensional Heat Conduction Problem
}

\author{
Ilmars KANGRO \\ Rezekne Academy of Technologies, Faculty of Engineering \\ Atbrivoshanas aleja 115, Rezekne, LV-4601, Latvia \\ ilmars. kangroerta. Iv
}

\begin{abstract}
The article focuses on the tasks of the mathematical physics - one-dimensional diffusion-convection boundary-value problem (BVP) for solving the heat conduction equation with piece-wise smooth coefficients in the multi-layer media. For this purpose the conservative averaging method (CAM) is using with special created integral splines of exponential type that interpolate the middle integral values of piece-wise smooth function through averaging in $\mathrm{z}$ direction. Thus BVP is reduced to the system of ordinary differential equations (ODE) dependent on time - this enables to find out the averaged solutions of BVP - non-stationary and stationary.
\end{abstract}

Keywords: 1-D diffusion-convection initial-boundary value problem, conservative averaging method, exponential type splines

\section{Introduction}

The numerical modelling of mathematical physics 1-D problems in layered medium using engineering-technical calculations of sufficient accuracy is important in numerous areas of the applied sciences.

Therefore we are studying the conservative averaging method (CAM) by using special integral exponential type splines with parameters in every layer, which means that the values of these parameters have to be chosen to decrease the error of approximation of the solution.

In the limit case when parameters tend to zero we have the integral parabolic type spline, developed by A. Buikis (Buikis, 1994a; Buikis,1994b).

CAM can be applied both to linear processes (Kalis, 2016) and non-linear processes (the dependency of mathematical model equation coefficients on the process characteristics, such as temperature in the combustion process) (Aboltins, 2017), (Weber, 2012). 


\section{Formulation of the problem}

The non-stationary diffusion-convection problem is studied in 1-D domain $\Omega=\left\{(z): 0 \leq z \leq L_{z}\right\}$.

The domain $\Omega$ consists of $N$-layered medium. We will consider the non-stationary 1-D problem of the linear diffusion theory for layered piece-wise homogenous materials of one $(N=1)$ and two $(N=2)$ layers.

$$
\Omega_{i}=\left\{(z): z \in\left(z_{i-1}, z_{i}\right)\right\}, i=\overline{1, N},
$$

where $H_{i}=z_{i}-z_{i-1}$ is the height of layer $\Omega_{i}, z_{0}=0, z_{N}=L_{z}$.

We can find the distribution of concentrations $u_{i}=u_{i}(z, t)$ in every layer $\Omega_{i}$ at the point $(z)=\Omega_{i}$ and at the time $t$ by solving the following initial-boundary value problem for partial differential equation (PDE):

$$
\left\{\begin{array}{l}
\frac{\partial u_{i}}{\partial t}=\frac{\partial}{\partial z}\left(D_{i z} \frac{\partial u_{i}}{\partial z}\right)+r_{i z} \frac{\partial u_{i}}{\partial z}-a_{i 0}^{2} u_{i}+F_{i}(z), z \in\left(z_{i-1}, z_{i}\right), t \in\left(0, t_{f}\right], i=\overline{1, N} \\
\gamma_{1 z} \partial u_{1}(0, t) / \partial z-\beta_{z}\left(u_{1}(0, t)-C_{0 z}\right)=0, t \in\left[0, t_{f}\right] \\
\gamma_{2 z} \partial u_{2}\left(L_{z}, t\right) / \partial z+\alpha_{z}\left(u_{2}\left(L_{z}, t\right)-C_{a z}\right)=0, t \in\left[0, t_{f}\right] \\
u_{i}\left(z_{i}, t\right)=u_{i+1}\left(z_{i}, t\right), D_{i z} \frac{\partial u_{i}\left(z_{i}, t\right)}{\partial z}=D_{i+1, z} \frac{\partial u_{i+1}\left(z_{i}, t\right)}{\partial z}, t \in\left[0, t_{f}\right], i=\overline{1, N-1} \\
u_{i}(z, 0)=u_{i 0}, z \in\left[z_{i-1}, z_{i}\right], i=\overline{1, N}
\end{array}\right.
$$

where $\quad \gamma_{j z}>0,(j=1 ; 2)$,

$u_{i}=u_{i}(z, t)$ - concentrations functions in every layer,

$F_{i}, D_{i z}>0, C_{0 z}, C_{a z}, r_{i z}, a_{i 0}$ - constant coefficients,

$\alpha_{z}, \beta_{z} \geq 0$ - constant mass transfer coefficients,

$c_{a z}, c_{0 z}$ - the given concentration on the boundary for the boundary,

$t_{f}$ - the final time,

$u_{i 0}$ - the given initial condition.

It must be added, that in present paper a specific diffusion-convection process is investigated, for which the constancy of the source-function $F_{i}$ is inherent. For $N=1$ the conditions on the contact line are deleted. Similarly 3-D initial- boundary problem in $N=1$-layer domain is considered in (Kalis, 2016).

\section{The conservative averaging method (CAM) in z-direction using integral spline with two fixed exponential type functions}

Using CAM with respect to $z$ with fixed parametrical functions $f_{i z 1}, f_{i z 2}, i=\overline{1,2}$, we have 


$$
\begin{aligned}
& u_{i}(z, t)=u_{i z}(t)+m_{i z}(t) f_{i z 1}+e_{i z}(t) f_{i z 2}, \\
& \quad f_{i z 1}=\exp \left(a_{1 i z}\left(z-\bar{z}_{i}\right)\right)-\frac{2}{a_{1 i z} H_{i}} \sinh \left(0.5 a_{1 i z} H_{i}\right), \\
& f_{i z 2}=\exp \left(a_{2 i z}\left(z-\bar{z}_{i}\right)\right)-\frac{2}{a_{2 i z} H_{i}} \sinh \left(0.5 a_{2 i z} H_{i}\right), \\
& u_{i z}(t)=H_{i}^{-1} \int_{z_{i-1}}^{z_{i}} u_{i z}(z, t) d z-\text { the averaged values, } \int_{z_{i-1}}^{z_{i}} f_{i z 1}(z) d z=\int_{z_{i-1}}^{z_{i}} f_{i z 2}(z) d z=0, \\
& \bar{z}_{i}=\left(z_{i-1}+z_{i}\right) / 2, z \in\left[z_{i-1}, z_{i}\right], i=\overline{1,2} .
\end{aligned}
$$

For exponential functions we use following parameters:

$$
a_{1 i z}=-\frac{r_{i z}}{2 D_{i z}}-\sqrt{\frac{r_{i z}^{2}}{4 D_{i z}^{2}}+\frac{a_{i 0}^{2}}{D_{i z}}}, \quad a_{2 i z}=-\frac{r_{i z}}{2 D_{i z}}+\sqrt{\frac{r_{i z}^{2}}{4 D_{i z}^{2}}+\frac{a_{i 0}^{2}}{D_{i z}}}, i=\overline{1,2} .
$$

These parameters are the characteristic values of the solution of problem's (2.1) homogenous differential equation $\left(F_{i}=0\right)$ in the stationary case $\left(\partial u_{i} / \partial t=0\right)$. The parameters are also used by designing the stationary analytic solution of the above mentioned BVP.

Unknown functions $m_{i z}(t), e_{i z}(t)$ shall be determined from (2.1) applying boundary conditions by $z=0, z=L_{z}, z=z_{1}$.

$$
\begin{aligned}
& m_{1 z}(t)=m_{01}\left(u_{2 z}(t)-u_{1 z}(t)\right)+m_{11} e_{1 z}(t)+m_{12} e_{2 z}(t), \\
& m_{2 z}(t)=m_{02}\left(u_{2 z}(t)-u_{1 z}(t)\right)+m_{21} e_{1 z}(t)+m_{22} e_{2 z}(t), \\
& m_{01}=D_{2 z} a_{21}^{m} / s_{1}, s_{1}=D_{2 z} a_{21}^{m} d_{11}^{p}-D_{1 z} a_{11}^{p} d_{21}^{m}, m_{02}=m_{01} s_{2}, \quad s_{2}=a_{11}^{p} D_{1 z} /\left(a_{21}^{m} D_{2 z}\right), \quad m_{12}=D_{2 z}\left(a_{21}^{m} d_{22}^{m}-a_{22}^{m} d_{21}^{m}\right), \\
& m_{11}=\left(D_{1 z} a_{12}^{p} d_{21}^{m}-D_{2 z} a_{21}^{m} d_{12}^{p}\right) / s_{1}, \quad \text { where } \\
& m_{21}=m_{11} s_{2}+a_{12}^{p} D_{1 z} /\left(a_{21}^{m} D_{2 z}\right), \quad m_{22}=m_{12} s_{2}-a_{22}^{m} / a_{21}^{m}, \quad \\
& d_{i k}^{m}=\exp \left(-0.5 a_{k i z} H_{i}\right)-\frac{2}{a_{k i z} H_{i}} \sinh \left(0.5 a_{k i z} H_{i}\right), \\
& d_{i k}^{p}=\exp \left(0.5 a_{k i z} H_{i}\right)-\frac{2}{a_{k i z} H_{i}} \sinh \left(0.5 a_{k i z} H_{i}\right), a_{i k}^{m}=a_{k i z} \exp \left(-0.5 a_{k i z} H_{i}\right), \\
& a_{i k}^{p}=a_{k i z} \exp \left(0.5 a_{k i z} H_{i}\right), i, k=\overline{1,2} .
\end{aligned}
$$

The functions $e_{i z}(t)$ are in the form:

$$
e_{1 z}(t)=b_{11} u_{1 z}(t)+b_{12} u_{2 z}(t)+g_{3}, e_{2 z}(t)=b_{21} u_{1 z}(t)+b_{22} u_{2 z}(t)+g_{4} \text {, }
$$

where

$$
\begin{aligned}
& b_{11}=\left(d_{22}\left(\beta_{1}+b_{1}\right)-d_{12} b_{2}\right) / \operatorname{det}, b_{12}=\left(d_{12}\left(\alpha_{1}+b_{2}\right)-d_{22} b_{1}\right) / \operatorname{det}, \\
& b_{21}=\left(-d_{21}\left(\beta_{1}+b_{1}\right)+d_{11} b_{2}\right) / \operatorname{det}, b_{22}=\left(-d_{11}\left(\alpha_{1}+b_{2}\right)+d_{21} b_{1}\right) / \operatorname{det},
\end{aligned}
$$




$$
\begin{aligned}
& \beta_{1}=\beta_{z} / \gamma_{1 z}, \alpha_{1}=\alpha_{z} / \gamma_{2 z}, b_{1}=m_{01}\left(a_{11}^{m}-\beta_{1} d_{11}^{m}\right), b_{2}=m_{02}\left(a_{21}^{p}+\alpha_{1} d_{21}^{p}\right), \\
& g_{3}=-\left(C_{0 z} \beta_{1} d_{22}+C_{a z} \alpha_{1} d_{12}\right) / \operatorname{det}, g_{4}=\left(C_{0 z} \beta_{1} d_{21}+C_{a z} \alpha_{1} d_{11}\right) / \operatorname{det}, \\
& d_{11}=a_{11}^{m} m_{11}+a_{12}^{m}-\beta_{1}\left(d_{11}^{m} m_{11}+d_{12}^{m}\right), d_{12}=m_{12}\left(a_{11}^{m}-\beta_{1} d_{11}^{m}\right), \\
& d_{21}=m_{21}\left(a_{21}^{p}+\alpha_{1} d_{21}^{p}\right), \\
& d_{22}=a_{21}^{p} m_{22}+a_{22}^{p}+\alpha_{1}\left(d_{21}^{p} m_{22}+d_{22}^{p}\right), \operatorname{det}=d_{11} d_{22}-d_{12} d_{21} .
\end{aligned}
$$

The method of CAM is applied - we integrate the equation of the system (2.1) by variable $z$ within the boundaries of each layer, and divide it by each layer's height $H_{i}$ then we insert function (3.1) and use the system (2.1) boundary conditions thus obtaining the system of ODEs (3.2):

$$
\begin{aligned}
& \left\{\begin{array}{l}
u_{1 z}^{\prime}(t)=c_{11} u_{1 z}(t)+c_{12} u_{2 z}(t)+g_{1} \\
u_{2 z}^{\prime}(t)=c_{21} u_{1 z}(t)+c_{22} u_{2 z}(t)+g_{2} \\
u_{1 z}(0)=u_{10}, u_{2 z}(0)=u_{20},
\end{array}\right. \\
& c_{11}=e_{11}\left(-m_{01}+m_{11} b_{11}+m_{12} b_{21}\right)+e_{12} b_{11}-a_{10}^{2} \text {, } \\
& c_{12}=e_{11}\left(m_{01}+m_{11} b_{12}+m_{12} b_{22}\right)+e_{12} b_{12} \text {, } \\
& c_{21}=e_{21}\left(-m_{02}+m_{21} b_{11}+m_{22} b_{21}\right)+e_{22} b_{21} \text {, } \\
& c_{22}=e_{21}\left(m_{02}+m_{21} b_{12}+m_{22} b_{22}\right)+e_{22} b_{22}-a_{20}^{2} \text {, } \\
& e_{11}=\frac{2}{H_{1}} \sinh \left(0.5 a_{11 z} H_{1}\right)\left(D_{1 z} a_{11 z}+r_{1 z}\right), e_{12}=\frac{2}{H_{1}} \sinh \left(0.5 a_{21 z} H\right)\left(D_{1 z} a_{21 z}+r_{1 z}\right) \text {, } \\
& e_{21}=\frac{2}{H_{2}} \sinh \left(0.5 a_{12 z} H_{2}\right)\left(D_{2 z} a_{12 z}+r_{2 z}\right), g_{1}=e_{11}\left(g_{3} m_{11}+g_{4} m_{12}\right)+e_{12} g_{3}+F_{1} \text {, } \\
& e_{22}=\frac{2}{H_{2}} \sinh \left(0.5 a_{22 z} H_{2}\right)\left(D_{2 z} a_{22 z}+r_{2 z}\right), g_{2}=e_{21}\left(g_{3} m_{21}+g_{4} m_{22}\right)+e_{22} g_{4}+F_{2} \text {. }
\end{aligned}
$$

The non-stationary solution of (3.2) can be represented in the following form:

$$
\begin{aligned}
& v(t)=\exp (A t)\left(v_{0}-A^{-1} F\right)+A^{-1} F, \quad A \text { is the matrix } \\
& A=\left(\begin{array}{cc}
c_{11} & c_{12} \\
c_{21} & c_{22}
\end{array}\right), \\
& v(t)=\left[u_{1 z}(t) ; u_{2 z}(t)\right], v_{0}=\left[u_{10} ; u_{20}\right], F=\left[F_{1} ; F_{2}\right] \text { are the vectors-columns. }
\end{aligned}
$$

The stationary averaged solution is

$$
u_{1 z}=\left(g_{2} c_{12}-g_{1} c_{22}\right) / d, u_{2 z}=\left(-g_{2} c_{11}+g_{1} c_{21}\right) / d, d=c_{11} c_{22}-c_{12} c_{21} \text {. }
$$

The stationary analytic solution is 
$\left\{\begin{array}{l}u_{1}(z)=P_{1} \exp \left(a_{11 z} z\right)+P_{2} \exp \left(a_{21 z} z\right)+f_{1} \\ u_{2}(z)=P_{3} \exp \left(a_{12 z} z\right)+P_{4} \exp \left(a_{22 z} z\right)+f_{2},\end{array}\right.$

$P_{1}=-\left(b_{1} P_{2}+b_{2}\right), P_{3}=b_{3} P_{4}-b_{4}, P_{2}=\left(b_{5} b_{22}+b_{6} b_{12}\right) / d, \quad P_{4}=\left(b_{6} b_{11}-b_{5} b_{21}\right) / d$,

$d=b_{11} b_{22}-b_{12} b_{21}, b_{1}=\frac{\beta_{1}-a_{21 z}}{\beta_{1}-a_{11 z}}, b_{2}=\frac{\beta_{1}\left(f_{1}-C_{0 z}\right)}{\beta_{1}-a_{11 z}}$,

$b_{3}=-\exp \left(L_{z}\left(a_{22 z}-a_{12 z}\right)\right) \frac{\alpha_{1}+a_{22 z}}{\alpha_{1}+a_{12 z}}, b_{4}=-\exp \left(-L_{z} a_{12 z}\right) \frac{\alpha_{1}\left(f_{2}-C_{a z}\right)}{\alpha_{1}+a_{12 z}}$,

$b_{5}=f_{2}-f_{1}+b_{2} \exp \left(a_{11 z} H_{1}\right)-b_{4} \exp \left(a_{12 z} H_{1}\right)$,

$b_{6}=b_{2} a_{11 z} \exp \left(a_{11 z} H_{1}\right)-b_{4} a_{12 z} k \exp \left(a_{12 z} H_{1}\right), k=\frac{D_{2 z}}{D_{1 z}}$,

$b_{11}=-b_{1} \exp \left(a_{11 z} H_{1}\right)+\exp \left(a_{21 z} H_{1}\right), b_{12}=b_{3} \exp \left(a_{12 z} H_{1}\right)+\exp \left(a_{22 z} H_{1}\right)$,

$b_{21}=-b_{1} a_{11 z} \exp \left(a_{11 z} H_{1}\right)+a_{21 z} \exp \left(a_{21 z} H_{1}\right)$,

$b_{22}=-k\left(b_{3} a_{12 z} \exp \left(a_{12 z} H_{1}\right)+a_{22 z} \exp \left(a_{22 z} H_{1}\right)\right), f_{1}=\frac{F_{1}}{a_{10}^{2}}, f_{2}=\frac{F_{2}}{a_{20}^{2}}$.

\section{The CAM in one layer}

In one layer we have following problem

$$
\left\{\begin{array}{l}
\frac{\partial u}{\partial t}=\frac{\partial}{\partial z}\left(D_{z} \frac{\partial u}{\partial z}\right)+r_{z} \frac{\partial u}{\partial z}-a_{0}^{2} u+F_{0}, z \in\left(0, L_{z}\right), t \in\left(0, t_{f}\right] \\
D_{z} \partial u(0, t) / \partial z-\beta_{z}\left(u(0, t)-C_{0 z}\right)=0, t \in\left[0, t_{f}\right] \\
D_{z} \partial u\left(L_{z}, t\right) / \partial z+\alpha_{z}\left(u\left(L_{z}, t\right)-C_{a z}\right)=0, t \in\left[0, t_{f}\right] \\
u(z, 0)=u_{0}, z \in\left[0, L_{z}\right],
\end{array}\right.
$$

where $u=u(z, t)$ is the unknown function,

$D_{z}>0, \alpha_{z}, \beta_{z} \geq 0, F_{0}, C_{0 z}, C_{a z}, r_{z}, a_{0}$ are the constant coefficients.

Using averaged method with respect to $z$ we have

$$
\begin{aligned}
& u(z, t)=u_{z}(t)+m_{z}(t)\left(\exp \left(a_{1 z}\left(z-L_{z} / 2\right)\right)-\frac{2}{a_{1 z} L_{z}} \sinh \left(0.5 a_{1 z} L_{z}\right)\right)+ \\
& e_{z}(t)\left(\exp \left(a_{2 z}\left(z-L_{z} / 2\right)\right)-\frac{2}{a_{2 z} L_{z}} \sinh \left(0.5 a_{1 z} L_{z}\right)\right)
\end{aligned}
$$

where $u_{z}(t)=L_{z}^{-1} \int_{0}^{L_{z}} u_{z}(z, t) d z, \quad a_{1 z}=-\frac{r_{z}}{2 D_{z}}-\sqrt{\frac{r_{z}^{2}}{4 D_{z}^{2}}+\frac{a_{0}^{2}}{D_{z}}}$,

$$
a_{2 z}=-\frac{r_{z}}{2 D_{z}}+\sqrt{\frac{r_{z}^{2}}{4 D_{z}^{2}}+\frac{a_{0}^{2}}{D_{z}}} .
$$


The unknown functions $m_{z}(t), e_{z}(t)$ we obtain from boundary conditions (4.1):

1) For $z=0, D_{z}\left(m_{z} a_{1}^{m}+e_{z} a_{2}^{m}\right)=\beta_{z}\left(u_{z}-C_{0 z}+m_{z} d_{1}^{m}+e_{z} d_{2}^{m}\right)$,

2) For $z=L_{z}, D_{z}\left(m_{z} a_{1}^{p}+e_{z} a_{2}^{p}\right)+\alpha_{z}\left(u_{z}-C_{a z}+m_{z} d_{1}^{p}+e_{z} d_{2}^{p}\right)=0$,

where

$$
\begin{aligned}
& d_{k}^{m}=\exp \left(-0.5 a_{k z} L_{z}\right)-\frac{2}{a_{k z} L_{z}} \sinh \left(0.5 a_{k z} L_{z}\right), \\
& d_{k}^{p}=\exp \left(0.5 a_{k z} L_{z}\right)-\frac{2}{a_{k z} L_{z}} \sinh \left(0.5 a_{k z} L_{z}\right), \\
& a_{k}^{m}=a_{k z} \exp \left(-0.5 a_{k z} L_{z}\right), a_{k}^{p}=a_{k z} \exp \left(0.5 a_{k z} L_{z}\right), k=\overline{1,2}
\end{aligned}
$$

The functions $m_{z}(t), e_{z}(t)$ are in the following form:

$e_{z}(t)=-g u_{z}(t)+C_{a z} a+C_{0 z} b, m_{z}(t)=g_{1} u_{z}(t)+C_{a z} a_{1}+C_{0 z} b_{1}$, where

$g=\left(a_{11} \alpha_{1}+a_{21} \beta_{1}\right) /$ det $, \alpha_{1}=\alpha_{z} / D_{z}, \beta_{1}=\beta_{z} / D_{z}, b=\beta_{1} a_{21} /$ det,

$a=\alpha_{1} a_{11} / \operatorname{det}, g_{1}=\left(a_{22} \beta_{1}+a_{12} \alpha_{1}\right) / \operatorname{det}, b_{1}=-\beta_{1} a_{22} / \operatorname{det}, a_{1}=-\alpha_{1} a_{12} / \operatorname{det}$,

$a_{11}=a_{1}^{m}-\beta_{1} d_{1}^{m}, a_{21}=a_{1}^{p}+\alpha_{1} d_{1}^{p}, a_{12}=a_{2}^{m}-\beta_{1} d_{2}^{m}, a_{22}=a_{2}^{p}+\alpha_{1} d_{2}^{p}$,

$\operatorname{det}=a_{11} a_{22}-a_{12} a_{21}$.

We integrate the equation of the system (4.1) by variable $z$ between $0, L$, and divide it by layer height $L$ then we insert function (4.2) and use the system (4.1) boundary conditions thus obtaining an initial value problem (4.3) for the ODE:

$$
u_{z}^{\prime}(t)=a_{2} u_{z}(t)+g_{2}, u_{z}(0)=u_{0},
$$

where $a_{2}=d_{1} g_{1}-d_{2} g-a_{0}^{2}<0, g_{2}=F_{0}+d_{1}\left(b_{1} C_{0 z}+a_{1} C_{a z}\right)+d_{2}\left(b C_{0 z}+a C_{a z}\right)$, $d_{1}=\frac{2}{L_{z}} \sinh \left(0.5 a_{1 z} L_{z}\right)\left(D_{z} a_{1 z}+r_{z}\right), d_{2}=\frac{2}{L_{z}} \sinh \left(0.5 a_{2 z} L_{z}\right)\left(D_{z} a_{2 z}+r_{z}\right)$.

Then, the non-stationary averaged solution is $u_{z}(t)=\left(u_{0}-g_{2} / a_{2}\right) \exp \left(a_{2} t\right)+g_{2} / a_{2}$.

The stationary averaged solution is $u_{z}=g_{2} / a_{2}$.

The stationary analytical solution is

$u(z)=P_{1} \exp \left(a_{1 z} z\right)+P_{2} \exp \left(a_{2 z} z\right)+f_{1}$,

where $P_{1}=b_{1} P_{2}+b_{2}, P_{2}=\frac{-b_{2} \exp \left(a_{1 z} L_{z}\right)\left(a_{1 z}+\alpha_{1}\right)+\alpha_{1}\left(C_{a z}-f_{1}\right)}{b_{1} \exp \left(a_{1 z} L_{z}\right)\left(a_{1 z}+\alpha_{1}\right) \exp \left(a_{2 z} L_{z}\right)+\left(a_{2 z}+\alpha_{1}\right)}$,

$b_{1}=\left(a_{2 z}-\beta_{1}\right) /\left(\beta_{1}-a_{1 z}\right), b_{2}=\left(C_{0 z}-f_{1}\right) /\left(\beta_{1}-a_{1 z}\right), f_{1}=f_{0} / a_{0}^{2}$. 


\section{Some numerical results}

The results of calculations are obtained by MATLAB. We use the discrete values

$x_{j}=j h, j=\overline{0, N_{x}}, N_{x} h=L_{z}=3, t_{n}=n \tau, n=\overline{0, N_{i}}, N_{t} \tau=t_{f}=19$,

$N_{t}=100, N_{x}=20, u_{0}=0, \alpha_{z}=100, \beta_{z}=0.0001, C_{a z}=1, C_{0 z}=5$.

For two layers: $F_{1}=0.6, F_{2}=0.4, r_{1 z}=-0.001, r_{2 z}=0.001, a_{10}=0.6, a_{20}=0.4$,

$D_{1 z}=0.1, D_{2 z}=0.01, \gamma_{1 z}=0.1, \gamma_{2 z}=0.01, H_{1}=1.8, H_{2}=1.2$,

$a_{11 z}=-1.892, a_{21 z}=1.902, a_{12 z}=-4.050, a_{22 z}=3.950$.

For one layer: $F_{0}=0.5, D_{z}=0.01, r_{z}=0.01, a_{0}=0.5, a_{1 z}=-5.52, a_{2 z}=4.52$.

In the following Figs. 1-3 there are represented the numerical and analytical (for stationary problem) results obtained by CAM using exponential type splines and "pdepe" for one and two layers. MATLAB routine "pdepe" solves nonlinear PDEs of the following form $(u=u(z, t))$ (WEB, a):

$$
c\left(z, t, u, \frac{\partial u}{\partial z}\right) \frac{\partial u}{\partial t}=\frac{\partial}{\partial z}\left(f\left(z, t, u, \frac{\partial u}{\partial z}\right)\right)+s\left(z, t, u, \frac{\partial u}{\partial z}\right),
$$

where $f$ is a flux term and $s$ is a source term. For all $t$ and either $z=z l=0$ or $z=z r=L_{z}$, the solution components satisfy two boundary conditions of the form

$$
p(z, t, u)+q(z, t) f\left(z, t, u, \frac{\partial u}{\partial z}\right)=0
$$

The error of approximation for stationary solutions with exponential type spline is $10^{-7}$, with parabolic type spline (Buikis, 1994b) - 0.212 (Figure 1, b)), for non-stationary solution with exponential type splines -0.016 (Figure2, a)). For one layer the maximal error of approximation for non-stationary solution is 0.018 (Figure2, b)).

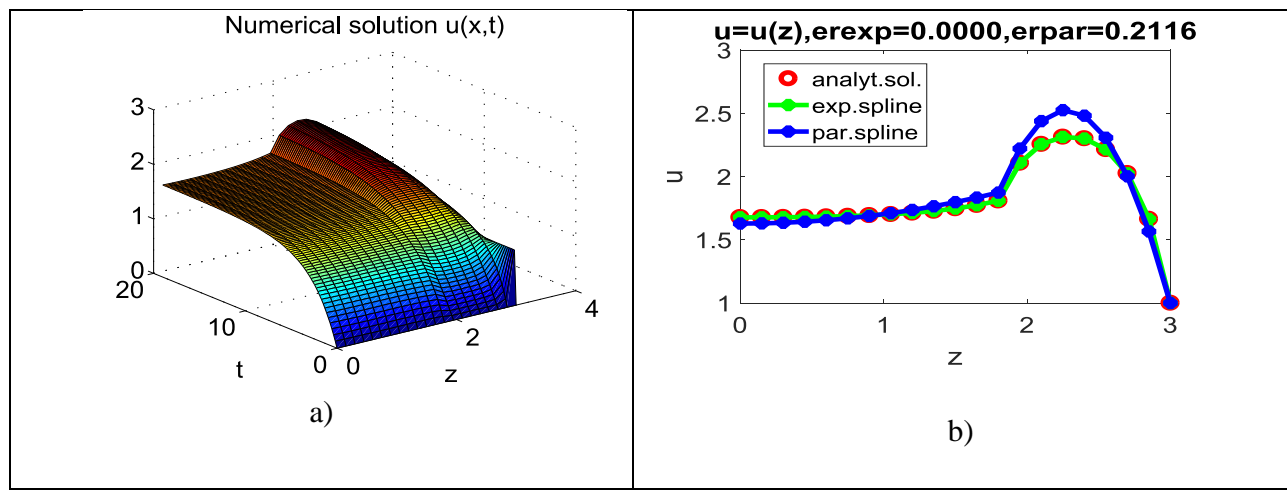

Figure1. Surface of solution generated by "pdepe" (a), stationary solution (analytical, generated by exponential and parabolic splines) (b) 


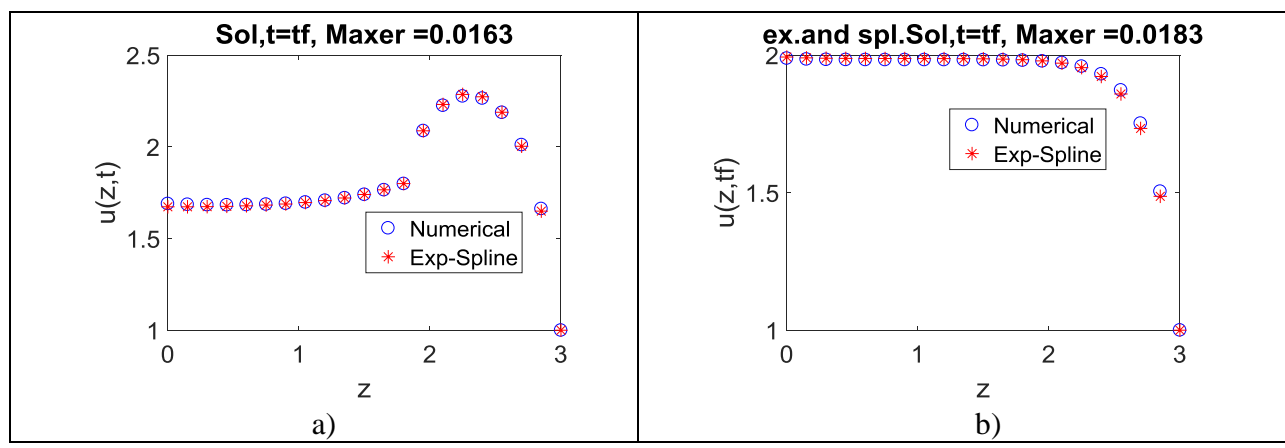

Figure2. Solution generated by "pdepe" and by exponential splines, $\mathrm{t}=\mathrm{t}_{\mathrm{f}}$ :

a) for two layers; b) for one layer

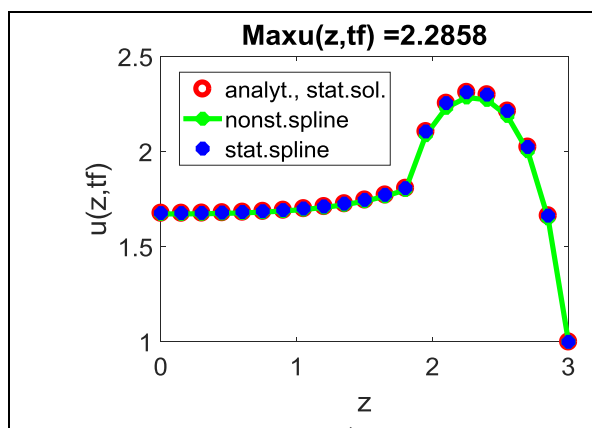

a)

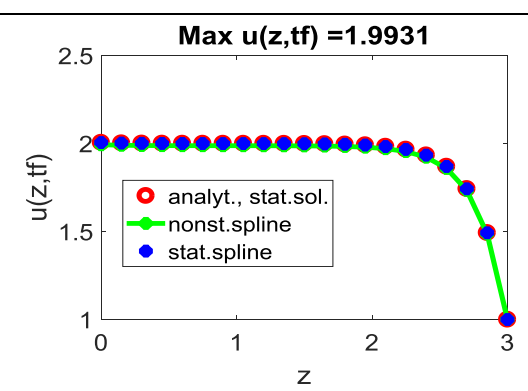

b)

Figure3. Stationary solution (analytical and generated by exponential spline)

and non-stationary generated by exponential spline, $t=t_{f}$ : a) for two layers; $b$ ) for one layer

\section{Conclusions}

The 1-D non-stationary diffusion-convection problem in a layered domain applying the conservative averaging method (CAM) is reduced to initial value problem (IVP) of ODEs using the created integral exponential type splines with two different functions each of them contain the parameter.

The error of approximation using the splines depends on these parameters. It was established that, to obtain a minimal error of approximation, the parameters of spline function must be equal to characteristic values of the solution of homogenous ODEs for the above mentioned IVP.

The stationary problems are solved analytically but the solutions of corresponding averaged non-stationary initial-boundary-value problems are obtained numerically also applying MATLAB routine "pdepe". 
The numerical solutions are compared with the analytical solutions and the matching results can be considered sufficiently accurate for engineering-technical calculations.

The third-type boundary conditions used in the mathematical model allow for the modelling of studied processes (filtration, combustion) in the direction of the flow changes of their characteristics (substance temperature, concentration, humidity, etc.).

It must be added that the CAM can be also used to solve more complex 3-D problems of mathematical physics by initially reducing them to 2-D problems and then solving with the method described herein.

\section{References}

Aboltins, A., Kalis, H., Pulkis, K., Skujans, J., Kangro, I. (2017). On Mathematical Modelling of Heat Transfer problem for a two layered gypsum board products exposed to fire. 16th International Scientific Conference Engineering for Rural Development, May 24-26, 2017, Jelgava, Latvia, Volume 16, pp.1369-1376., Elsevier SCOPUS:

DOI:10.22616/ERDev2017.16.N312, (ISSN: 1691-5976).

Buikis, A. (1994a). The analysis of schemes for the modelling same processes of filtration in the underground. Riga, Acta Universitatis Latviensis, 592, 1994, 25-32 (in Latvian).

Buikis, A. (1994b). The approximation with splines for problems in layered systems. Riga, Acta Universitatis Latviensis, 592, 1994, 135-138 (in Latvian).

Kalis, H., Buiķis, A., Kangro, I. (2016). Special Splines of Exponential Type for the Solutions of Mass Transfer Problems in Multilayer Domains. Mathematical Modelling and Analysis, Volume: 21, Issue: 04, pages 450 -465. Elsevier SCOPUS: (http://dx.doi.org/10.3846/13926292.2016.1182594), (ISSN: 1392-6292)

Weber, B. (2012). Heat transfer mechanisms and models for a gypsum board exposed to fire. International Journal of Heat and Mass Transfer, 55 2012, 1661-1678.

WEB (a). PDE Initial-Boundary Value Problem Solver.

Retrieved April 1, 2018, from https://se.mathworks.com/

Received June 26, 2018, accepted June 26, 2018 